\title{
Role of urinary CTX I in early diagnosis of osteoarthritis
}

\begin{abstract}
Introduction: The early diagnosis of osteoarthritis is required to do possible intervention in early stage of disease because it is one of the leading causes of disability in advance stage. For this reason various biomarker have been studied having merits and demerits of each. We chose urinary CTX-1 as biomarker to assess its role in osteoarthritis in knee joint.

Methodology: the study included 100 patients of primary osteoarthritis of knee joint and 50 controls. They were subjected to WOMAC scoring and K-L grading and estimation of urinary CTX-I. Statistical analysis was done using SPSS software version 20.0.

Results: Mean CTX-I levels of Control Group were 12.28 $\pm 5.3 \mathrm{ng} / \mathrm{dl}$ and in Case Group was $37.13 \pm 23.8 \mathrm{ng} / \mathrm{dl}$ and this difference was highly significant $(\mathrm{p}<0.001)$. Strong correlation was found between K-L grading and urinary CTX-I on Spearman's correlation coefficient. These associations were found to be highly significant $(\mathrm{p}<0.001)$. Receiver operator curve (ROC) analysis was done for prediction of cut-off values between Control group and Case group found $>14.4 \mathrm{ng} / \mathrm{ml}$ as cut-off point between Control group and Case group. Conclusion: Since CTX-I is biomarker of bone resorption and bone resorption is found more in case of progressive osteoarthritis of knee joint hence, we believe that CTX I levels can help in early identification of progressive OA cases.
\end{abstract}

Keywords: CTX I, knee osteoarthritis, progressive osteoarthritis, K-L grading, JSW, type1 collagen
Volume 3 Issue I - 2015

\author{
Sudhir Singh,' Dharmendra Kumar, ${ }^{2}$ Rahul \\ Raja' \\ 'Department of Orthopaedics, Era Medical College, India \\ ${ }^{2}$ Department of Orthopaedics, KGMU, India
}

\begin{abstract}
Correspondence: Sudhir Singh, Head - Department of Orthopaedics, Era's lucknow medical college, Lucknow, India, Tel 8799544905, Email susi59@live.in
\end{abstract}

Received: June 09, 2015 | Published: June 25, 2015
Abbreviations: ROC, receiver operator curve; CTX-I, carboxy cross-linked telopeptide type-I; OA, osteoarthritis; ASA, acetylsalicylic acid; ELISA, enzyme linked immuno-sorbent assay; BMD, bone mineral density

\section{Introduction}

The characteristic of osteoarthritis $(\mathrm{OA})$ is cartilage loss that leads to joint destruction and severe impairment of mobility. The bestestablished methods of assessing the progression of cartilage loss are measurement of the joint-space width on plain radiography, and arthroscopic evaluation of the knee. ${ }^{1}$ To visualize changes in jointspace width requires a couple of years before it can be detected and by that time much cartilage damage usually occurs. ${ }^{2,3}$ Arthroscopy is an invasive technique that cannot be routinely applied to all patients. Hence, to diagnose osteoarthritis in early stage different biomarker has been studied to do possible intervention to prevent further damage.

As OA affects mainly subchondral bone, cartilage and the synovium hence structural molecules derived from these tissues can be candidate biological markers for OA. Several studies have suggested that subchondral bone structure is altered in patients with osteoarthritis. ${ }^{4-6}$ Degradation product of type 1 collagen can be measured in urine as pyridinoline cross-links and their excretion is considerably high in osteoarthritis. ${ }^{4}$ We have taken urinary carboxy cross-linked telopeptide type-I collagen (CTX I) as a biomarker in patients with osteoarthritis of knee joint because it's release into blood is earlier than biomarkers of cartilage turnover. ${ }^{7,8}$

\section{Materials and methods}

A case control study was conducted in the Department of Orthopaedics and Microbiology in ERA'S Lucknow Medical College \& Hospital, Lucknow for 18 months. The study was approved by
Institutional research and ethics committee. The recruited subjects (cases and controls) were explained the purpose and relevance of the study and those who volunteered were included in the study after informed and written consent. In this study a total of 150 subjects between the age of 40 and 85 years were included out of which there were 100 cases of knee osteoarthritis and 50 normal healthy subjects were included in control group. All patients with signs and symptoms pertaining to primary knee osteoarthritis reporting to Orthopaedics OPD were screened and those who fit into the clinical criteria of primary knee osteoarthritis as per American college of rheumatology were included in this study. ${ }^{9}$ All healthy subjects between 40 and 85 years of age with no signs and symptoms of Osteoarthritis and preferably first degree relatives of the cases were taken as controls. Patients were excluded if they had:

1. Secondary osteoarthritis,

2. Drug treatment for osteoarthritis or any other disease,

3. Active renal, hepatic diseases and malignancy,

4. History of alcohol or drug abuse,

5. Sensitivity to diclofenac, acetylsalicylic acid (ASA) or any other NSAIDs, acetaminophen, dimethyl sulphoxide, propylene glycol, glycerin or ethanol,

6. Pregnancy and lactating mothers,

7. Any other pathology affecting the knee and

8. Been an athletic or sportsperson in recent past.

After enrolling the subject demographic data like age, gender, height, weight, body mass index (BMI) and WOMAC score was recorded in a proforma and a detailed history was taken and clinical 
examination done. Antero-posterior and lateral view radiographs of both knee in weight bearing standing position, laboratory test and urinary analysis of CTX-I by ELISA (enzyme linked immuno-sorbent assay) Kit manufactured by Qayee-Bio, Shanghai, China, was done. Kellgren-Lawrence grading (K-L Grading) of the X-rays was done. The assessment of severity was done on the basis K-L grades and the case group was subdivided as mild grade (K-L Grade II), moderate grade (K-L grade III) and severe grade (K-L grade IV) of the disease. Normal healthy subjects with no signs and symptoms of osteoarthritis of knee and having K-L grade 0 and K-L grade I were labeled as controls.

Urinary sample of the recruited cases and controls were collected in a sterile container and 5mlurinary sample was kept in a plain vial taking all aseptic precautions. The samples taken were kept at room temperature before sending in to laboratory. The urine was centrifuged for 20 minutes at 2000-3000 rpm and stored in small capped vials for long term use at $-200 \mathrm{C}$ until tested. Urinary CTX-I was measured by the enzyme linked immunosorbent assay (ELISA).

\section{Statistical analysis}

Data was collected and statistically analyzed using SPSS software version 20.0. Frequency, percentage, mean, standard deviation, correlation, one way ANOVA test and ROC curves was used to present the data; p-values $<0.05$ were considered statistically significant. Independent $t$ test and chi square was used to assess the difference between the two study groups.

\section{Observation and results}

A total of 150 subjects (100 cases and 50 controls) were enrolled in this study. The demographic data of the patients under study is shown in Table 1. In Control group there were 22 males and 28 females and in Case group there were 36 males and 64 females. The mean age was $56.17 \pm 11.4$ years (range 40-81), mean weight was $60.17 \pm 7.2 \mathrm{~kg}$ (range 50-85), mean height was $160.22 \pm 9.5 \mathrm{cms}$ (range 145-185) and mean BMI $\left(\mathrm{kg} / \mathrm{m}^{2}\right)$ was $23.44 \pm 1.9$ (range 20.32 - 27.63). The mean of duration of disease in years was $2.85 \pm 1.8$ (range $0.2-7.0$ ).

Table I Shows demographic data of different parameters of both groups

\begin{tabular}{|c|c|c|c|c|c|c|}
\hline $\begin{array}{l}\text { Demographic } \\
\text { data }(\mathbf{N}=150)\end{array}$ & & Range & $\begin{array}{l}\text { Total subjects } \\
(\mathrm{N}=\mid 50)\end{array}$ & $\begin{array}{l}\text { Control } \\
(\mathrm{N}=50)\end{array}$ & $\begin{array}{l}\text { Cases } \\
(\mathrm{N}=100)\end{array}$ & P Value \\
\hline Age (Year) & & $40-85$ & $56.17 \pm 11.4$ & $55.12 \pm 11.2$ & $56.70 \pm 11.6$ & 0.427 \\
\hline \multirow[t]{2}{*}{ Sex } & Male (\%) & 58 & $58(38.7 \%)$ & 22 (37.9\%) & $36(62.1 \%)$ & 0.343 \\
\hline & Female (\%) & 92 & $92(61.3 \%)$ & 28 (30.4\%) & 64 (69.6\%) & \\
\hline Height $(\mathrm{cm})$ & & $145-185$ & $160.22 \pm 9.5$ & $160.9 \pm 10.8$ & $159.8 \pm 8.7$ & 0.48 \\
\hline Weight (kg) & & $50-85$ & $60.17 \pm 7.2$ & $61.5 \pm 7.5$ & $59.5 \pm 7.0$ & 0.12 \\
\hline BMI $\left(\mathrm{kg} / \mathrm{m}^{2}\right)$ & & $20.32-27.63$ & $23.44 \pm 1.9$ & $23.8 \pm 2.0$ & $23.31 \pm 1.8$ & 0.16 \\
\hline
\end{tabular}

\section{$\mathrm{N}$, sample size; $\mathrm{SD}$, standard deviation}

Number of patients in different K-L grades in Cases Group is given in Table 2 . In case group, $22 \%$ cases had grade II $60 \%$ cases had grade III and $18 \%$ cases had grade IV K-L grading. Mean WOMAC score

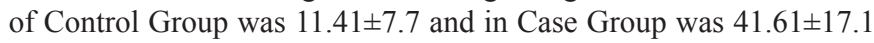

and this was statistically highly significant $(\mathrm{p}<0.001)$. Mean CTX-I levels of Control Group were 12.28 $\pm 5.3 \mathrm{ng} / \mathrm{dl}$ and in Case Group was $37.13 \pm 23.8 \mathrm{ng} / \mathrm{dl}$ and this difference was highly significant $(\mathrm{p}<0.001)$.

Table 2 Comparison of K-L Grade,WOMAC score and serum CTX I levels in controls and cases

\begin{tabular}{|c|c|c|c|c|}
\hline & & \multicolumn{2}{|l|}{ Mean $\pm S D$} & \multirow{2}{*}{ Statistical significance } \\
\hline & & Control (I & Cases $(N=100)$ & \\
\hline & Grade II & 0 & $22(22 \%)$ & \\
\hline \multirow[t]{2}{*}{$\mathrm{K} / \mathrm{L}$ grade } & Grade III & 0 & $60(60 \%)$ & $\mathrm{X}^{2}=\mathrm{a} P=\mathrm{a}$ \\
\hline & Grade IV & 0 & $18(18 \%)$ & \\
\hline \multicolumn{2}{|c|}{ WOMAC Score } & || $.4 \mid \pm 7.7$ & $41.61 \pm 17.1$ & $t=-11.895, p=<0.001$ \\
\hline \multicolumn{2}{|c|}{ CTX I Level (ng/ml) } & $12.28 \pm 5.3$ & $37.13 \pm 23.8$ & $\mathrm{t}=-7.279, \mathrm{p}=<0.001$ \\
\hline
\end{tabular}

$\mathrm{N}$, sample size; SD, standard deviation; ng/ml, nanogram per milliliter; Grade 2: mild; Grade 3: moderate; Grade 4: severe; a: No statistics are computed because Group is a constant

Correlation coefficient of CTX I was calculated for age, WOMAC score and K-L grade (Table 3). CTX-I level showed a mild association of with age $(\mathrm{r}=0.358)$ and WOMAC score $(\mathrm{r}=0.420)$ and strong association with K-L grade $(r=0.691)$. These associations were found Table 3 Correlation coefficient in different Age, K-L grade and WOMAC score (Spearman's correlation coefficient)

\begin{tabular}{llll}
\hline & $\begin{array}{l}\text { Correlation coefficient } \\
\text { (pearson's correlation coefficient - "r") }\end{array}$ & PValue & $\begin{array}{l}\text { Power of } \\
\text { correlation }\end{array}$ \\
\hline Age & 0.358 & $<0.001$ & Mild \\
K-L grade & 0.691 & $<0.001$ & Strong \\
WOMAC Score & 0.420 & $<0.001$ & Mild \\
\hline
\end{tabular}

to be highly significant $(\mathrm{p}<0.001)$. CTX-I levels and WOMAC scores in various severity grades of osteoarthritis in Case group is shown in Table 4.

\author{
.
}


Table 4 comparison of severity of primary knee osteoarthritis and CTX I levels

\begin{tabular}{llll}
\hline Severity & N & $\begin{array}{l}\text { CTX-I level } \\
\text { Mean } \pm \text { SD }\end{array}$ & $\begin{array}{l}\text { WOMAC score } \\
\text { Mean } \pm \text { SD }\end{array}$ \\
\hline Mild & 22 & $19.38 \pm I 1.9$ & $31.82 \pm 8.3$ \\
Moderate & 60 & $40.41 \pm 22.32$ & $40.22 \pm 15.6$ \\
Severe & 18 & $47.90 \pm 28.77$ & $58.23 \pm \mid 8.5$ \\
Total & 100 & $37.13 \pm 23.8$ & $41.61 \pm \mid 7.1$ \\
Significance & & $\mathrm{F}=10.092, \mathrm{p}=<0.001$ & $\mathrm{~F}=16.090, \mathrm{p}=<0.001$
\end{tabular}

Receiver operator curve (ROC) analysis was done based on the direction of assessment and CTX-I level was evaluated for prediction of cut-off values between Control group and Case group (Table 5 \& Figure 1). The results suggested a cut-off value of $>14.4 \mathrm{ng} / \mathrm{ml}$ between Control group and Case group.

Table 5 Showing accuracy of cut-off values of CTX I as suggested by ROC Curve

Area under the curve

test result variable(s): CTX-I level(ng/ml)

\begin{tabular}{ll}
\hline & $\begin{array}{l}\text { Asymptotic 95\% Confidence } \\
\text { Area Std. Error }{ }^{\mathrm{a}} \text { Asymptotic Sig. }\end{array}$ \\
interval \\
Lower bound Upper bound
\end{tabular}

$0.9110 .025 \quad 0.000 \quad 0.862 \quad 0.96 \mid$

A. Under the nonparametric assumption. b. Null hypothesis: true area $=0.5$

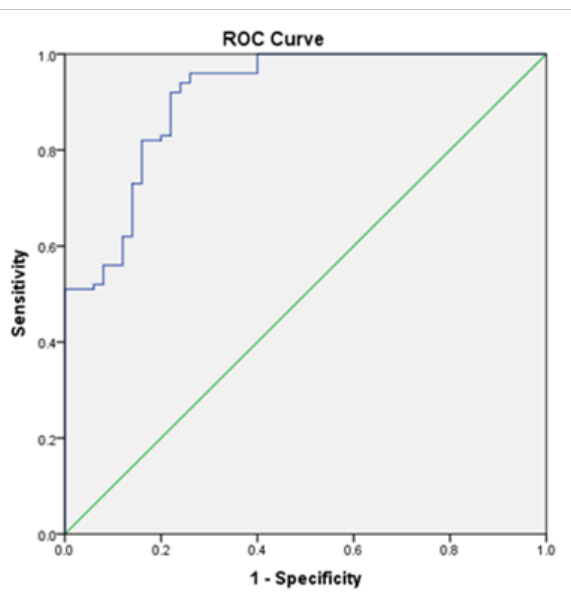

Figure I Showing ROC curve for CTX IValues between control and cases.

\section{Discussion}

$\mathrm{OA}$ is one of the most prevalent causes of disability in the aging population. The best-established methods of assessing the progression of cartilage loss are measurement of the joint-space width on plain radiography, and arthroscopic evaluation of the knee. ${ }^{1}$ Changes in joint-space width are, however, relatively small compared with the precision error of radiographic measurements, and currently serial assessments over 2-3 years are required to obtain reliable information on the progression of joint damage or its retardation as a result of treatment. MRI is more sensitive than plain radiography, but the validity of MRI for monitoring patients with OA is currently being assessed. ${ }^{10}$ Arthroscopy, though provides a direct and magnified view of the cartilage surface, but is an invasive technique that cannot be routinely applied to all patients. Joint space width assessed as K-L grade though remains gold standard to diagnose OA but it neither gives early diagnosis nor efficient monitoring of efficacy of treatment can be done. ${ }^{7}$ Given the limitations of the tools that are currently available for investigation of OA, it is not surprising that there has been considerable interest in the identification of specific biological markers that reflect quantitative and dynamic variations in joint remodelling. ${ }^{7}$

As OA affects mainly bone, cartilage and the synovium, it follows that structural molecules derived from these tissues could be candidate biological markers for OA. In recent years, different biochemical markers of bone turnover have been developed and validated in humans and in animal models. ${ }^{11}$ Present study, the first on Indian Population, was carried out with an objective to ascertain the role of urinary analysis of CTX-I (Carboxy Cross linked Telopeptide Type-I Collagen) in early diagnosis and assessment of severity of knee osteoarthritis. We selected CTX I as a biomarker as usefulness of CTX I estimation in diagnosing progressive OA has been reported by many authors. ${ }^{4}$ Streich NA ${ }^{12}$ reported increase in CTX I level is related with increase in local production of inflammatory cytokines resulting in uncoupling of bone turnover and finally cartilage degradation. ${ }^{12}$ Increased levels of urinary CTX I indicates increased bone resorption in cases with progressive OA but not in non-progressive OA has been reported earlier. ${ }^{7,8,13,14}$ Garnero $\mathrm{P}$ et al. ${ }^{15}$ reported that high baseline urinary CTX I independently predicts an increased risk of OA. ${ }^{15}$ In patients with hip OA urinary CTX I levels indicate higher cartilage and bone turnover has been reported by Takahashi M. ${ }^{16}$ Davis $\mathrm{CR}^{17}$ reported correlation of CTX I levels with K-L grades and joint space width and hence reflects general pathological changes in knee. ${ }^{17}$ Garnero P et al. ${ }^{18}$ reported that CTX I is release follows CTX II just as bone degradation follows cartilage destruction. ${ }^{18}$ But few recent studies have also reported no association of severity of knee OA with CTX I and Osteocalcin levels. ${ }^{19,20}$

The demographic profile of subjects like age, height, weight and BMI in Case group and Control group was similar having statistically significant difference between them ( $p>.05)$. The number of female subject was higher in Case group and control group as well but this difference was not statistically significant (Table 1). All control group subjects had K-L grade 0 or grade I. Both, WOMAC score and CTX I levels were significantly higher in Cases group than in Control group (Table 2). Correlation of urinary CTX I levels with age, WOMAC score and K-L grade was calculated. Age $(\mathrm{r}=0.358)$ and WOMAC score $(\mathrm{r}=0.420)$ both showed only mild correlation with urinary CTX I levels though showed a significant correlation $(<0.001)$. This is similar to what has been reported by Garnero P et al. ${ }^{19}$

But K-L grade $(\mathrm{r}=0.691)$ showed a strong correlation with CTX I levels (Table 3). This signifies the fact though CTX I is basically a marker for bone turnover yet it has a strong and significant correlation $(<0.001)$ to the cartilage degradation as measured by plain radiography and depicted as K-L grading. CTX-I levels of Cases and Control subjects showed increasing trends with increasing grades of K-L Grades (Table 4). Correlation of CTX I with K-L grading has been shown by other authors as well. ${ }^{18}$ But in a similar study Tanishi $\mathrm{N}$ et al. ${ }^{21}$ did not find any significant difference between the urinary CTX1 levels in the different OA grades (K-L grade). ${ }^{21}$ But the authors also reported that the higher quartiles of urinary CTX1 levels gradually included higher numbers of K-L grade $\geq$ II OA subjects in the over 60 year-old women. But another study reported a similar observation as in present study. ${ }^{22}$ 
CTX-I levels and WOMAC scores were found to be higher for higher grades of severity and this difference was statistically significant $(p<0.001)$. Similarly, WOMAC scores also showed a significant increasing trend from doubtful to severe knee OA cases $(p<0.001)$. The Receiver operator curve $(\mathrm{ROC})$ analysis was done and it suggested a cut-off value of CTX-I level as $>14.4 \mathrm{ng} /$ $\mathrm{ml}$ between Control group and Case group. This cut-off value was highly sensitive $(96.0 \%)$ and specific $(74.0 \%)$ to differentiate between early osteoarthritis cases and from normal asymptomatic population with an accuracy of $91.1 \%$. Presently there is no study on this issue for comparison. Present study has several limitations that merit discussion. Small sample size restricts application of these results on a larger general population globally. A much larger multicentric study with subjects of different geographical region and race would be needed to do this accurately and replicate the results. Secondly, since CTX I is bone resorption biomarker hence co-existing concomitant osteoporosis can be a possible confounding factor. Hence, we believe that in future studies to be done along with simultaneous bone mineral density (BMD) measurements and with bone a formation biomarker level assessment as well. Analysis of results of urinary CTX I levels in OA subjects with normal BMD and those with low BMD might give more accurate outcome.

\section{Conclusion}

This pilot study showed a mild association of age $(\mathrm{r}=0.358)$ and WOMAC score $(\mathrm{r}=0.420)$ with CTXI level which is highly significant $(p<0.001)$. A strong association between CTX I level and K-L Grade $(r=0.691)$ was found which again was statistically highly significant $(\mathrm{p}<0.001)$. CTX I level was evaluated for prediction of cut-off values between Control group and Case group by ROC curve. The results suggested a cut-off value of $>14.4 \mathrm{ng} / \mathrm{ml}(96.0 \%$ sensitive and $74.0 \%$ specific with accuracy of $91.1 \%$ ) between Control group and Case group. Since bone resorption is only increased in patients with progressive knee OA and not in those whose knee OA does not progress, hence we believe that CTX I levels can help in early identification of progressive OA cases.

\section{Acknowledgements}

None.

\section{Conflicts of interest}

The authors declare there is no conflict of interest.

\section{References}

1. Blackburn WD, Chivers S, Bernreuter W. Cartilage imaging in osteoarthritis. Semin Arthritis Rheum. 1996;25(4):273-281.

2. Petersson IF, Jacobsson LT. Osteoarthritis of the peripheral joints. Best Pract Res Clin Rheumatol. 2002;16(5):741-760.

3. Kirwan JR, Elson CJ. Is the progression of osteoarthritis phasic? Evidence and implications. J Rheumatol. 2000;27(4):834-836.

4. Stewart A, Black A, Robins SP, et al. Bone density and bone turnover in patients with osteoarthritis and osteoporosis. $J$ Rheumatol. 1999;26(3):622-626.

5. Bettica P, Cline G, Hart DJ, et al. Evidence for increased bone resorption in patients with progressive knee osteoarthritis: longitudinal results from the Chingford study. Arthritis Rheum. 2002;46(12):3178-3184.
6. Dieppe P, Cushnaghan J, Young P, et al. Prediction of the progression of joint space narrowing in osteoarthritis of the knee by bone scintigraphy. Ann Rheum Dis. 1993;52(8):557-563.

7. Garnero P, Rousseau JC, Delmas PD. Molecular basis and clinical use of biochemical markers of bone, cartilage, and synovium in joint diseases. Arthritis Rheum. 2000;43(5):953-968.

8. Bettica P, Cline G, Hart DJ, et al. Evidence for increased bone resorption in patients with progressive knee osteoarthritis: longitudinal results from the Chingford study. Arthritis Rheum. 2002;46(12):3178-3184.

9. Altman R, Asch E, Bloch D, et al. Development of criteria for the classification and reporting of osteoarthritis, classification of osteoarthritis knee. Arthritis Rheum. 1986;29(8):1039-1049.

10. Gandy SJ, Dieppe PA, Keen MC, et al. No loss of cartilage volume over three years in patients with knee osteoarthritis as assessed by magnetic resonance imaging. Osteoarthritis and Cartilage. 2002;10(12):929937.

11. Calvo MS, Eyre DR, Gundberg CM. Molecular basis and clinical application of biological markers of bone turnover. Endocr Rev. 1996;17(4):333-368.

12. Streich NA, Zimmermann D, Schmitt H, et al. Biochemical markers in the diagnosis of chondral defects following anterior cruciate ligament insufficiency. Int Orthop. 2011;35(11):1633-1637.

13. Rovetta G, Monteforte P, Grignolo MC, et al. Hematic levels of type I collagen C-Telopeptide in erosive versus nonerosive osteoarthritis of the hands. Int J Tissue React. 2003;25(1):25-28.

14. Berry PA, Maciewicz RA, Cicuttini FM, et al. Markers of bone formation and resorption identify subgroups of patients with clinical knee osteoarthritis who have reduced rates of cartilage loss. J Rheumatol. 2010;37(6):1252-1259.

15. Garnero P, Landewe R, Boers M, et al. Association of baseline levels of markers of bone and cartilage degradation with long-term progression of joint damage in patients with early rheumatoid arthritis: the COBRA study. Arthritis Rheum. 2002;46(11):2847-2856.

16. Takahashi M, Suzuki M, Naitou K, et al. Comparison of free and peptide-bound pyridinoline cross-links excretion in rheumatoid arthritis and osteoarthritis. Rheumatology (Oxford). 1999;38(2):133-138.

17. Davis CR, Karl J, Granell R, et al. Can biochemical markers serve as surrogates for imaging in knee osteoarthritis? Arthritis Rheum. 2005;56(12):4038-4047.

18. Garnero P, Mazieres B, Gueguen A, et al. Cross-sectional association of 10 molecular markers of bone, cartilage and synovium with disease activity and radiological joint damage in patients with hip osteoarthritis: the ECHODIAH cohort. J Rheumatol. 2005;32(4):697-703.

19. Garnero P, Piperno M, Gineyts E, et al. Cross sectional evaluation of biochemical markers of bone, cartilage, and synovial tissue metabolism in patients with knee osteoarthritis: relations with disease activity and joint damage. Ann Rheum Dis. 2001;60(6):619-626.

20. Jordan KM, Syddall HE, Garnero P, et al. Urinary CTX-II and glucosyl-galactosyl-pyridinoline are associated with the presence and severity of radiographic knee osteoarthritis in men. Ann Rheum Dis. 2006;65(7):871-877.

21. Tanishi N, Yamagiwa H, Hayami T, et al. Usefulness of urinary CTX-II and NTX-I in evaluating radiological knee osteoarthritis: the Matsudai knee osteoarthritis survey. J Orthop Sci. 2014;19(3):429-436.

22. Garnero P. Biochemical markers of bone remodeling. Annales de Biologie Clinique. 2001;59(3):298. 